inferred from the most subtle differences of light and shade in the colours of objects, in association with previous experience derived through other senses. The picture is always an optical illusion; and this additional illusiveness conferred on the photograph by the method invented by Maxwell on the basis of the three-colour theory of vision, is surely a strong confirmation of that theory.

These results are attained by no new photographic operations. It is necessary to use good orthochromatic plates sensitised into the red, and also to have affixed in the lens an orthochromatic screen cutting of the ultra-violet light in the usual manner. The exposure is somewhat longer than the ordinary exposure, for we can of course only use visible light, and of this a part is stopped by the taking screen. The ordinary backs may be used. The displacement of the sensitive film from accurate register with the ground glass camera screen, owing to the presence of the taking screen in front of it, may be corrected (if thought necessary) by simply reversing the surface of the ground glass camera screen, turning the muffled side outward. This secures that the image will be accurately focussed in the plane of the sensitive surface. Negatives and positives may be used as ordinary negatives or positives till it is desired to recall the original colours. Thus, for those who wander with the camera, the possession of but the one seeing screen to test results is sufficient, and of course the one taking screen suffices to take an indefinite number of plates.

These considerations lead us naturally to observe that the registration of colour being really carried in the silver image, which with very little care in manipulation may be made permanent, secures that the colours are permanent. A faded screen may at any time be made good by a fresh screen; the colours in all cases being spectroscopically chosen, we are assured of the reproduction of the original colour. In this aspect the necessity of the detached colour screen is no disadvantage, but rather a necessary safeguard against the inevitable fading attending most pigment colours.

\section{COMET MAGNITUDES.}

D. HOLETSCHEK, of the Vienna Observatory, has recently communicated to the Imperial Academy of Sciences a paper on the magnitude and brilliancy of comets and their tails, with the view of arranging them in "magnitudes" or orders of brilliancy in a manner similar to that in which stars are arranged according to their lucidity. Further, from the data given as to the apparent length of the tail, the true length of the tail has been computed, and an inquiry instituted as to the possibility of tail formation and its probable length, based on the resulting magnitude of the comet and its perihelion distance.

If it be true that the brilliancy of a comet varies as the squares of the distances from the sun $(r)$, and from the earth $(\Delta)$, then from observations made at various points of the orbit, the same "magnitude" ought to result for the values $r=\Delta=\mathrm{I}$. This magnitude Dr. Holetschek has deduced, where sufficient data existed, and the results can be practically arranged in two classes : one, in which the deduced magnitudes derived from various values of $r$ and $\Delta$ so nearly agree that a mean can be taken ; the other, in which is shown a regular progress, and always in the direction that the deduced magnitudes with small radii vectures, therefore when the comet is near perihelion, are greater than when at large distances from the sun. The origin of this is due to the fact that the second power of the radius vector does not fully represent the variations occurring in comets as they approach the sun, at which time their brilliancy is more increased than is shown by the ratio I : $r^{2} \Delta^{2}$. The first case, it is suggested, is only a special case of the second, arising through insufficiency of description. This is most clearly shown when the time covered by the observations is short, or the details so wanting in accuracy that the deviation from the ratio $\mathrm{I}: r^{2} \Delta^{2}$ cannot be known with certainty. The conclusion drawn is that the formula so generally used can represent the brilliancy of a comet at different distances from the sun for a short time only, and is inapplicable for long periods.

Dr. Holetschek uses the deduced magnitude in the neighbourhood of the perihelion, valuable as showing the greatest brilliancy attainable in a particular comet, as the data for forming the comets into orders of magnitude, and inquires how far the tail formation is connected with this magnitude and the perihelion distance. He decides from his material, that when the deduced NO. I $36 \mathrm{I}$, VOL. 53 ] magnitude is 6 or lower than 6 , only a short and feeble tail, or one not visible to the naked eye, is possible. Comets with a de. duced magnitude of 4 , or still brighter, have a tail well visible, which is the greater, the smaller the perihelion distance, and the smaller, the greater this distance. Within the limits between 4 and 5 magnitude, if we exclude very great perihelion distances, lie the possibilities of a considerable tail development.

Dr. Holetschek has also considered the diameters of comets, and sought to introduce order by reducing the apparent diameter to that corresponding to a distance of the comet from the earth equal to unity. In the case of Halley's comet, no diminution or variability is to be detected in either its brilliancy or the length of its tail. The same values serve from 1456 to 1835 . As, however, on account of the continual development of the tail, a diminution of the mass is probable, it cannot be decided whether the approximate constancy, shown in the investigation, arises from inadequacy in the observations themselves, or is produced by certain processes existing in cometary bodies.

\section{UNIVERSITY AND EDUCATIONAL INTELLIGENCE.}

OxForD.-The delegates of the Common University Fund have elected Mr. Edwin Stephen Goodrich, Merton College, to the Biological Scholarship at Naples for the year I895-96.

CAMBRIDGe.-The report to the Senate of the Engineering Laboratory Syndicate, dated November 9, 1895, is a very satisfactory record of energetic work. The University was unable to provide more than $£ 1000$ for the building and equipment of the laboratory, which was estimated to cost about 6600. By steady and enthusiastic efforts Prof. Ewing and his colleagues succeeded in collecting from many sources, within and without the University, the $£ 5000$ that were needed; and what is still more to their credit, have completed the work in hand with a small balance to the good. The continued growth of the department, in which there are now eighty-five students under instruction, makes a further extension of the buildings urgently necessary. A site has been provided for the purpose, but further funds are needed for construction. The workshops, never intended to be other than temporary, must soon be rebuilt, and more lecture-room accommodation must be provided. The department has certainly justified its existence, and the Syndicate have proved themselves to be worthy stewards of the funds placed at their disposal. It is to be hoped that, with these guarantees that they will be well used, the needful moneys may ere long be forthcoming. The valuable services of Mr. Dalby and Mr. Lamb, the demonstrators of mechanism and engineering, in the work of organising the laboratory, are mentioned with cordial appreciation in the report. Among the donors of contributions of over $£ 100$ are the Duke of Devonshire, the late Earl of Derby, Mr. Frank McClean, and Dr. John Hopkinson, and eleven benefactors have given $f$ ioo each. Valuable donations of apparatus and of books have helped greatly towards the furnishing of the laboratory.

The amount, clear of all expenses, available for the Robertson Smith memorial, is $£_{1450}$. It has been agreed by the subscribers that sufficient of this amount should be invested to produce an annual income of $f 30$, such income to be employed on the continuance and extension of Prof. Robertson Smith's library, which he bequeathed to Christ's College. It was also resolved that the remainder of the amount collected, after all expenses have been paid, be handed over to the University for the purchase of Oriental MSS. for the University Library, which shall be marked as having been acquired by means of the fund. It is estimated that about $£ 300$ will be handed over to the Syndics of the Library.

IT is reported that Mr. P. N. Russell has given the sum of $£ 50,000$ to endow a school of engineering in connection with the Sydney University.

AN address on the present state and position of technical in struction in this country, delivered by Major-General Sir John Donnelly before the Society of Arts on Wednesday, November 20 , is printed in full in the current Fournal of the Society.

The annual meeting of the National Association for the Promotion of Technical and Secondary Education, and the Conference of Representatives of Technical Education Committees, will be held on Tuesday, December Io, at the Royal United Service Institution, Whitehall. 